\title{
STARK BROADENING OF STELLAR Pt II LINES
}

\author{
MILAN S. DIMITRIJEVIĆ \\ Astronomical Observatory, Volgina 7, 11050 Belgrade, Yugoslavia
}

Lines of Pt II have been discovered in Hg Mn stars by Dworetsky (1969). The analysis of a few strong Pt II transitions, which are also observed in IUE spectra of stars, has shown (Dworetsky et al., 1984) "that $\mathrm{Pt}$ is, like $\mathrm{Hg}$, among the most overabundant elements in the atmospheres of $\mathrm{Hg} \mathrm{Mn} \mathrm{stars,}$ with enhancements of the order of $10^{4}$ to $10^{5}$ over the solar system abundances". Dworetsky et al. (1984) selected also the four Pt II lines which might be used for astrophysical applications. Moreover, they determined the corresponding theoretical $g f$ values. The aim of this contribution is to investigate the Stark broadening of these $\mathrm{Pt}$ II lines and to provide the corresponding Stark widths.

In the case of more complex atoms or multiply charged ions the lack of accurate atomic data needed for more sophisticated calculations diminishes the reliability of the semiclassical results. In such cases approximate methods might be very interesting. Good possibilities provide e.g. the modified semi-empirical method (Dimitrijević and Konjević, 1980), which have been used here for these calculations. Our results for four Pt II lines selected by Dworetsky et al. (1984) as the most interesting ones from an astrophysical point of view, are presented in Table 1 . In order to see the influence of the differences in oscillator strengths, results of calculations with $g f$ values obtained by using the Coulomb approximation are presented as well. The differences might give an impression of the error bars in the obtained results.

We can also see from Table 1 that all lines belong to the same supermultiplet and that their widths are not very different. Using the analysis of Stark broadening parameters within a supermultiplet (Dimitrijević, 1982) we can estimate the Stark widths of other members within multiplets and the supermultiplet using $\mathrm{W}_{1}=\left(\lambda^{2}{ }_{1} / \lambda^{2}{ }_{2}\right) \mathrm{W}_{2}$, taking for $\mathrm{W}_{2}$ the most appropriate value for the considered case (i.e. e.g. the nearest available member of the same multiplet).

For an order of magnitude estimate, we might use the above mentioned equation, taking for $W_{2}$ the data for the transition with the same upper level (or the nearest available member of the same multiplet). For optical Pt II lines given in Dworetsky et al. (1984) and Dworetsky and Vaughan (1973), we might scale by this relation the $\lambda=2245.5 \AA$ data for the $\lambda=$ $4148.30 \AA, 4061.66 \AA, 4034.17 \AA, 4023.81 \AA$ and $3447.78 \AA$ lines; the $\lambda=$ $1781.9 \AA$ data for the $3806.91 \AA$ line and the $\lambda=2144.2 \AA$ data for the $\lambda$ $=4514.17 \AA, 4288.40 \AA, 4046.45 \AA, 3766.40 \AA$, and $3577.20 \AA$ lines. 


\section{TABLE 1}

Full Stark widths in $\AA$ of astrophysically important Pt II lines as a function of temperature $T$ in $\mathrm{K}$. The electron density is $10^{17} \mathrm{~cm}^{-3}$. The Stark width $W_{1}$ has been calculated by using oscillator strengths of Dworetsky et al. (1984) and $W_{2}$ with oscillator strengths calculated with the Coulomb approximation.

\begin{tabular}{lrrrl}
\hline Transition & $\lambda(\AA)$ & $T(\mathrm{~K})$ & $W_{1}(\AA)$ & $W_{2}(\AA)$ \\
\hline Pt II $6 \mathrm{~s}^{4} \mathrm{~F}_{9 / 2}-6 \mathrm{p}^{4} \mathrm{G}_{11 / 2}$ & 1777.1 & 5000 & 0.0496 & 0.0353 \\
& & 10000 & 0.0351 & 0.0249 \\
& & 20000 & 0.0248 & 0.0176 \\
& & 40000 & 0.0176 & 0.0125 \\
& 80000 & 0.0124 & 0.00882 \\
Pt II $6 \mathrm{~s}^{4} \mathrm{~F}_{7 / 2}-6 \mathrm{p}^{4} \mathrm{G}_{9 / 2}$ & 2245.5 & 5000 & 0.0529 & 0.0557 \\
& & 10000 & 0.0374 & 0.0394 \\
& & 20000 & 0.0264 & 0.0279 \\
& & 40000 & 0.0187 & 0.0197 \\
Pt II $6 \mathrm{~s}^{4} \mathrm{~F}_{9 / 2}-6 \mathrm{p}^{4} \mathrm{~F}_{9 / 2}$ & 1781.9 & 50000 & 0.0132 & 0.0139 \\
& & 5000 & 0.0502 & 0.0358 \\
& & 20000 & 0.0355 & 0.0253 \\
& & 40000 & 0.0177 & 0.0127 \\
Pt II $6 \mathrm{~s}^{4} \mathrm{~F}_{9 / 2}-6 \mathrm{p}^{4} \mathrm{D}_{7 / 2}$ & 2144.2 & 5000 & 0.0692 & 0.0484 \\
& & 10000 & 0.0489 & 0.0342 \\
& & 20000 & 0.0346 & 0.0242 \\
& & 40000 & 0.0245 & 0.0171 \\
& & 80000 & 0.0174 & 0.0122 \\
\hline & & & 0.0179 \\
& & &
\end{tabular}

\section{References}

Dimitrijević, M.S.: 1982, Astronomy and Astrophysics 112, 251

Dimitrijević, M.S., Konjević, N.: 1980, JQSRT 24, 451

Dworetsky, M.M.: 1969, Astrophysical Journal 156, L101

Dworetsky, M.M., Storey, P.J., Jacobs, J.M.: 1984, Physica Scripta T8, 39

Dworetsky, M.M., and Vaughan, Jr.H.: 1973, Astrophysical Journal 181, 811 